\title{
CLASES DE PALABRAS Y PREDICACIÓN. REFLEXIONES PARA LA ENSEÑANZA
}

María Mare

\section{(c) $(7) \Theta$}

Doi: https://doi.org/10.15517/rfl.v46i1.41117

URL: https://revistas.ucr.ac.cr/index.php/filyling/index 



\title{
CLASES DE PALABRAS Y PREDICACIÓN. REFLEXIONES PARA LA ENSEÑANZA
}

\author{
WORD CLASSES AND PREDICATION. \\ REFLECTIONS ON TEACHING
}

María Mare

\begin{abstract}
RESUMEN
Dos temas que recorren la escolarización -visibilizados en planes de estudios y manuales de lengua- son clases de palabras y predicación. Ambas nociones suelen asociarse fundamentalmente al contenido léxico, lo que lleva a confusiones importantes, no solo por la arbitrariedad y la heterogeneidad de las definiciones dadas, sino por la dificultad para establecer generalizaciones pertinentes. El abordaje de la sintaxis desde una perspectiva formal y a partir del empleo de un metalenguaje adecuado es fundamental para promover la reflexión gramatical entre nuestros estudiantes. Este tipo de acercamiento al estudio del lenguaje es necesario para entender que la clase de lengua no es un espacio de reproducción de normas lingüísticas o repetición de conceptos, sino que puede convertirse en un laboratorio científico. El objetivo de este trabajo es presentar algunas discusiones centrales que debemos considerar los docentes de lengua para promover el pensamiento crítico y el desarrollo de habilidades lingüísticas en los diferentes niveles educativos.

Palabras clave: enseñanza de la gramática; estructura argumental; clases de palabras; diversidad lingüística; metalenguaje.
\end{abstract}

\begin{abstract}
There are two topics which are found throughout education, as can be seen in language syllabi and textbooks: word classes and predication. Both concepts are usually related to lexical meaning, which creates great confusion not only owing to the arbitrariness and heterogeneity of the definitions taught in class, but also to the difficulty in reaching suitable generalizations. A formal approach to syntax that takes into account a proper use of metalanguage is necessary to promote grammar discussion among our students. This particular approach to language lets us understand language lessons as a scientific laboratory, instead of a moment of repetition of concepts and normative rules. The goal of the present paper is to develop some theoretical concerns that language teachers should revisit so as to promote critical thinking and to develop linguistic skills in different levels of education.

Keywords: grammar teaching; argumental structure; word classes; linguistic diversity; metalanguage.
\end{abstract}

Dra. María Mare. Profesora adjunta de la Universidad Nacional del Comahue. Investigadora asistente, Instituto Patagónico de Estudios de Humanidades y Ciencias Sociales, Consejo Nacional de Investigaciones Científicas y Técnicas (IPEHCS-CONICET). Río Negro, Argentina.

Correo electrónico: maria.mare@fahu.uncoma.edu.ar

Recepción: 07- 05- 19

Aceptación: 18- 06- 19 


\section{Introducción}

En la curricula escolar, tanto de Argentina como de otros países hispanohablantes (y de otros países, en general), es posible reconocer contenidos específicos de gramática oracional, independientemente de si el foco está puesto en mayor o menor medida en las funciones del lenguaje ${ }^{1}$. Sin embargo, a pesar de que estos contenidos están contemplados, su abordaje en el aula no es del todo claro y su enseñanza aparece muchas veces de manera forzada, en un contexto en el que no resulta evidente su pertinencia. Si pensamos en los manuales escolares, no es difícil encontrar una unidad en la que se presenta un texto sobre las tradiciones argentinas, por ejemplo, y el hecho de que en ese texto haya sustantivos -como en cualquier otro- parece servir como excusa para habilitar la enseñanza de esta clase de palabras. Las definiciones y actividades que se proponen son, muchas veces, limitadas y, por esta razón, terminan enfrentando el conocimiento de nuestros estudiantes como hablantes nativos de una lengua con el tipo de conocimiento que provee el manual y el docente de turno. Por ejemplo, ya que fue mencionada la clase de los sustantivos, de la que hablaré en este texto, recordemos lo que se suele enseñar sobre sustantivos propios: "se escriben con mayúscula", "nombran seres o lugares en particular", "no van precedidos por artículo". Cualquiera de estos enunciados entra inmediatamente en conflicto con nuestro conocimiento como hablantes nativos del español: ¿cómo identifico la mayúscula en la oralidad?; ¿mamá y casa se escriben con minúscula, pero nombran seres y lugares en particular?; ¿por qué mamá y casa son sustantivos comunes si los puedo usar sin artículo?; ¿en mi familia utilizamos el artículo para hablar de la gente conocida: mi vecina es la Rosa, mi hermano es el Marcos?

Ante este conflicto hay dos posibles caminos: obviar que nuestros estudiantes tienen conocimientos como hablantes nativos e inhabilitar toda discusión posible, con las consecuencias que esto conlleva (ver Carrió, 2014, por ejemplo) o bien, no solo recuperar esos conocimientos, sino promover el cuestionamiento a las definiciones dadas a partir de los datos de las variedades que conviven en el aula. Este segundo recorrido propicia no solo la investigación, sino también la reflexión sobre la argumentación gramatical (Mare, 2014). Para lograr esto, es fundamental que, como docentes de lengua, comencemos haciéndonos preguntas: ¿qué enseñamos en la clase de lengua?; ¿para qué enseñar sintaxis o, de manera más amplia, para qué enseñar gramática?; ¿qué queremos que nuestros alumnos aprendan?

Los objetivos de este artículo son los siguientes: revisar algunos conceptos a través de los cuales se aborda la descripción de las lenguas; promover el estudio reflexivo de la gramática y discutir distintas estrategias didácticas para el abordaje de los contenidos propuestos. Como indiqué anteriormente, me centraré en las clases de palabras y en la predicación por tres motivos. En primer lugar, porque como ya mencioné son los contenidos que están presentes a lo largo de toda la escolarización; en segundo lugar, porque están estrechamente relacionados; y, en tercer lugar, porque lo que refiere a la predicación, de manera más general, a la estructura argumental, es central para la formación de oraciones, y de manera más global, de cualquier texto. Como señala Di Tullio (2005, p. 103), "el predicado es como el guión de una obra: determina cuántos participantes se requieren y asigna a cada uno un papel".

La organización de los apartados es la siguiente. Luego de esta introducción, en §2 presento algunos conceptos que resultan centrales para definir qué enseñamos en la clase

1 Ver, por ejemplo, el diseño curricular del Ministerio de Educación, Ciencia y Tecnología, Núcleos de aprendizajes prioritarios (2006), Argentina. 
de lengua; en el apartado 3, me detendré en la noción de "clase de palabra", para analizar y discutir los criterios de clasificación; en $\$ 4$ se aborda la predicación, la estructura argumental y la relación entre argumentos y funciones sintácticas. En cada uno de estos apartados explicitaré posibles actividades vinculadas a las reflexiones propuestas. Finalmente, en $\$ 5$ se exponen las conclusiones.

\section{Empezando desde el principio}

Uno de los problemas con los que se enfrenta la gramática es la idea que existe con respecto a sus objetivos. Lamentablemente, la mirada normativista ha tenido un peso muy fuerte en la escuela y en la sociedad, en general, y resulta una tarea extremadamente compleja erradicar ciertos presupuestos que desencadenan un sinnúmero de prejuicios lingüísticos sobre las distintas variedades y ponen al gramático en el lugar de quien define qué es hablar bien y qué es hablar mal. Por este motivo, es fundamental considerar las dimensiones desde las que se puede "evaluar" una determinada construcción lingüística. Me detendré aquí en los conceptos de gramaticalidad, corrección y adecuación (Bosque y Gutiérrez-Rexach, 2009; Di Tullio, 2005). Observemos las oraciones de (1).
a. Re pifió la.
b. ¿Vistes que no me eligieron?
c. El chabón tiró la posta.

La respuesta general frente a estas oraciones es indicar que están todas mal y sugerir la opción que se considera buena. Cuando se intenta indagar un poco más, los planteos suelen quedarse en el plano comunicativo: "lo que quiso decir fue...". Evidentemente, ninguna de estas respuestas permite avanzar demasiado en el conocimiento de la lengua y en discusiones de índole gramatical. Sin embargo, al detenernos un poco más, empiezan a surgir diferencias entre "nadie dice esto" - para evaluar (1a), por ejemplo-, "la gente lo dice, pero está mal" para definir una oración como (1b) y "eso se dice entre amigos" - con respecto a (1c). Estos tres tipos de evaluaciones que realizamos los hablantes son centrales para el desarrollo de la investigación lingüística en el aula de lengua, siempre y cuando, como docentes propongamos avanzar en la discusión con nuevas preguntas, en lugar de asumir que una frase como "la gente lo dice, pero está mal" constituye una respuesta relevante.

Veamos cómo se relacionan estas consideraciones con respecto a los enunciados de (1) con las nociones que se utilizan en la labor de investigación. El enunciado "nadie dice esto" se vincula estrechamente con el concepto de gramaticalidad, que se propone para indicar si una determinada secuencia se ajusta a los principios que articulan el sistema al que pertenece. Así, por ejemplo, la forma rompido en lugar de roto constituye una secuencia gramatical, porque se construye a partir de las reglas del sistema: los participios pasivos del español se forman regularmente a partir de la sufijación del morfema -do a la base verbal (canta- > cantado; come-> comido; parti-> partido). Es decir, rompido surge a partir de la aplicación inconsciente de los principios que subyacen a la formación de participios del español. Roto debe aprenderse como una excepción a esa regla. En cambio, una construcción como "re pifió la" en (1a) es imposible en español, porque presenta un orden que no se forma a partir de los 
principios del sistema, de ahí que ningún hablante diga esta expresión ${ }^{2}$. En este sentido, se considera agramatical. Si indagamos con nuestros estudiantes la agramaticalidad de este dato, que necesariamente es un dato "construido" para los fines de la reflexión gramatical, podemos avanzar en discusiones interesantes con respecto al orden de constituyentes en español: posición de los cuantificadores / intensificadores (la re pifió vs. la pifió $\underline{\mathrm{mal}}$ ), ubicación de clíticos como la (la pifió vs. pifiarla), entre muchísimas cosas que podemos decir sobre esta construcción y sobre las formas gramaticales, que muestran una regularidad y productividad sorprendentes (la re pifió, la embarró, la rompió, etc.).

Un aspecto interesante es que el concepto de gramaticalidad permite reconocer sistemas lingüísticos diferentes. Es claro que el inglés y el español tienen gramáticas distintas, pero en ciertos ámbitos no es tan claro que a las diferencias entre el español que se habla en una zona y el que se habla en otra subyacen sistemas distintos. Por ejemplo, en la mayoría de las variedades del español, una oración como me acordé tuyo es agramatical, ya que los hablantes no producen esta forma, sino me acordé de vos / de tí. Sin embargo, en las variedades del español de Uruguay y Venezuela (ver Bertolotti, 2014; Mare, 2015), esta oración es gramatical y responde a las reglas del sistema lingüístico de esas variedades. Así, incluir la noción de variedades lingüísticas en la clase de lengua es totalmente relevante, ya que en el aula conviven distintas variedades, y nuestros estudiantes, como hablantes nativos de estas, pueden realizar aportes valiosos para la discusión gramatical.

El otro enunciado para evaluar algunas de las construcciones de (1) es "la gente lo dice, pero está mal". A diferencia de lo que planteamos en el párrafo anterior, aquí no estamos hablando de datos construidos por los gramáticos, sino de construcciones que los hablantes efectivamente producen, pero que reciben algún tipo de sanción. Lo interesante es que si los hablantes emiten estas formas es porque los principios de su sistema lingüístico lo permiten. Intentar, entonces, entender las características de tales secuencias nos lleva necesariamente a hacer una investigación. La idea de "se dice pero está mal" se relaciona con el concepto de corrección, que refiere a factores externos al sistema lingüístico, motivados muchas veces por razones históricas o que resultan de determinadas relaciones sociales. Así, por ejemplo, rompido se sanciona como incorrecto y se propone roto como la forma correcta, que recupera una irregularidad ya presente en latín y que se conservó en la historia del español. Podemos decir, entonces, que ambas formas son gramaticales, ya que pertenecen al sistema lingüístico del español, pero que se diferencian en términos de corrección. En este plano, como dijimos, entra (1b), oración en la que aparece la forma verbal vistes, en lugar de la correcta viste. Si indicamos únicamente que vistes es incorrecto, no solo no avanzamos en la reflexión gramatical, sino que estamos señalando que quienes utilizan esta forma hablan mal y esto puede incluir a nuestros estudiantes y su familia.

Ahora bien, que los hablantes produzcan esta forma no es casual ni arbitrario. Quien dice vistes, agrega la $-s$ final no solo al verbo ver, sino a todos los pretéritos perfectos de la segunda persona del singular (cantastes, comistes, partistes). Aquí ya tenemos una generalización empírica, detrás de la cual, necesariamente subyace algún principio del sistema. Si miramos el resto del paradigma verbal correspondiente a la segunda persona del singular, reconocemos que las formas correspondientes terminan siempre en $-s$ (cantás, cantarás,

2 La forma gramatical es la re pifió, del verbo pifiar, que significa "equivocarse" (cometer una pifia). Re es un cuantificador característico del español rioplatense (ver Kornfeld y Kuguel, 2013). 
cantarías, cantes, etc.), excepto en el pretérito perfecto. Parecería, entonces, que a vistes subyace una regla propia del sistema: si el verbo concuerda con un sujeto de segunda persona del singular, termina en $-s$. El hecho de que esa forma sea evaluada como incorrecta es algo externo al sistema, algo que el hablante debe aprender no porque hable mal, sino porque en determinados contextos, la alternativa sin $-s$ final será la adecuada.

Veamos, entonces, la última noción que está en juego aquí y que se vincula con la frase "eso se dice entre amigos": la adecuación. Un ejemplo muy claro para referir a la adecuación es (1c), El chabón tiró la posta. Evidentemente, esta frase dicha entre amigos es perfecta, pero si la empleamos en un congreso para referir a las últimas reflexiones de Chomsky sobre el lenguaje, generará un efecto en el auditorio, ya que no es el tipo de expresión que se espera en un ámbito académico. Asimismo, si en un asado con amigos, alguien dice "estimados comensales, es mi intención informarles que desarrollaré una disertación sobre la elaboración de los choripanes", sus interlocutores se verán sorprendidos y asumirán este enunciado como algún tipo de broma, ya que resulta inadecuado para ese contexto. En relación al concepto de adecuación, aparece la noción de registro, que refiere a los diferentes modos de comportarse lingüísticamente. Así, la diferencia entre "el chabón tiró la posta” y "el disertante argumentó de manera convincente" podría pensarse en términos de registro si nos refiriéramos a las reflexiones de Chomsky. En el primer caso estamos ante un registro informal, mientras que el segundo corresponde a un registro formal. Ambos serán adecuados en un contexto, pero inadecuados en otro.

El docente de lengua debe ser capaz de plasmar en el aula el empleo de estos tres conceptos (gramaticalidad, corrección y adecuación), ya que son centrales para definir los objetivos de la clase de gramática y, en consecuencia, determinar qué corregir, cómo corregir y en qué momento hacerlo. Incluir la reflexión gramatical con respecto a las formas consideradas incorrectas no implica dejar de lado la enseñanza de las formas consideradas correctas. Es decir, si nuestros estudiantes atraviesan los doce años de escolarización y no incorporan otros recursos lingüísticos para indicar las habilidades expositivas de alguien, además de $E l$ chabón tiró la posta (expresión que seguramente incorporó por afuera del ámbito escolar), debemos reconocer que algo no está funcionando bien en el sistema educativo. Asimismo, si nuestros estudiantes se sienten estigmatizados por la variedad lingüística que adquirieron, que puede distar más o menos de la considerada "correcta", también debemos plantearnos si estamos haciendo las cosas bien en el aula. Por estas razones, creo que resulta fundamental que al momento de organizar un programa de lengua, los docentes consideremos las nociones presentadas en este apartado, tanto para definir los objetivos, como para delimitar los contenidos por desarrollar.

\section{Consideraciones sobre las clases de palabras}

En la introducción mencioné que la definición de sustantivo propio, que encontramos normalmente en los manuales y que muchas veces utilizan los docentes, recupera los siguientes aspectos: "se escriben con mayúscula", "nombran seres o lugares en particular", "no van precedidos por artículo". Vale la pena notar que cada una de estas afirmaciones pertenece a universos muy distintos. La primera responde a principios de normativa ortográfica, aplicables únicamente a la escritura. La segunda se enmarca en el plano del significado y la referencialidad. Finalmente, la tercera resulta de una descripción sintáctica, que se inscribe 
necesariamente en las reflexiones sobre los conceptos que presentamos en el apartado anterior: Rosa vino ayer vs. La Rosa vino ayer, ambas oraciones gramaticales, pero la segunda considerada incorrecta. Para alcanzar una descripción adecuada de los fenómenos del lenguaje, es fundamental reconocer los distintos criterios que están involucrados en las definiciones que utilizamos en nuestras prácticas de enseñanza. Y la pregunta que surge en relación con esto es: ¿por qué enseñamos contenidos que resultan de una complejidad extrema como si fueran obvios? ¿Por qué nos empeñamos en creer que decirles a nuestros estudiantes que el nombre propio no lleva artículo es suficiente para que puedan reconocer nombres propios? Veamos qué sucede cuando nos centramos en la forma y dejamos de lado el significado.

(2) Con mutipa, un brosqui pidrió umpa grasca y Murolla, a quien los poquis habían impipado, drinó la siquelición. Planimente, el brosqui que tataba de poritación tutucó a un cutú de la rutica. La rutica era sapalicosa, pero Zozó, el brosqui gudonte, no cucó. Aunque la rutica era zumú, le patiló la ratileza. Zozó, Murolla y fitos brosquis cafaron para lastrofar la matuca ${ }^{3}$.

Evidentemente, es posible reconocer clases de palabras como sustantivos (brosqui, grasca, Murolla, poquis, siquelición), verbos (pidrió, habían impipado, drinó, tataba) y adjetivos (sapalicosa, gudonte) aunque no sepamos si esas palabras designan cosas, acciones y cualidades, respectivamente. Parece claro, entonces, que el criterio semántico no resulta del todo pertinente para reconocer clases de palabras y, sin embargo, es al que más se recurre en las clases de lengua. Una objeción que se suele hacer a esta crítica es que el criterio semántico es más sencillo en ciertas edades que otros criterios formales y por eso es el que suelen recuperar los manuales y los docentes de lengua. Esta objeción es falaz en tres sentidos: (1) la filosofía ha demostrado que las discusiones sobre el significado constituyen un problema de enorme complejidad (ver, por ejemplo, las observaciones realizadas en Makuc Sierralta, 2010); (2) el criterio semántico atraviesa la escolaridad en los distintos años, sin distinguir entre el niño de siete años y el adolescente de dieciséis; y (3) las nociones formales vinculadas con la matemática presentan un grado de abstracción un tanto mayor que las reflexiones sobre la forma de la lengua materna de nuestros estudiantes: ¿cómo puede ser más sencillo aprender la regla de tres simple que reflexionar sobre la morfología de un sustantivo en español? Este mismo tipo de contradicciones se observa muchas veces en los colegios bilingües, en los que parece que en la clase de lengua extranjera los niños pueden realizar reflexiones gramaticales de forma, que no realizan con su lengua materna ${ }^{4}$.

El texto de (2) -o similares- se puede trabajar perfectamente en el aula de lengua, enfocando la atención de nuestros estudiantes de acuerdo con los objetivos de la clase. Si quisiéramos trabajar con la clase de los sustantivos, ya con la primera oración tenemos unas cuantas cosas interesantes para señalar. Indico en negrita las palabras que pueden clasificarse como sustantivos.

3 Este ejemplo está inspirado en un texto que figura en Serra y Caballer (1997). Realizamos esta adaptación para una de las capacitaciones docentes que dictamos en 2018 con el grupo denominado Gramática en el aula (Universidad Nacional del Comahue), en la que el foco de la discusión era la clasificación de sustantivos. Un ejemplo típico se da con respecto a la enseñanza de sustantivos contables y no contables. Esta distinción se aborda en las clases de inglés como lengua extranjera, pero cuando se sugiere trabajar esta clasificación en la clase de lengua en relación al español, muchos docentes platean que es un contenido muy complejo. 
(3) Con mutipa, un brosqui pidrió umpa grasca y Murolla, a quien los poquis habían impipado, drinó la siquelición.

Independientemente del grado de discusión y análisis que se proponga en la clase, el docente de lengua debe tener claro por qué las palabras en negrita pueden clasificarse como sustantivos y qué tipos de sustantivos podrían ser, de acuerdo con el contexto sintáctico. Empecemos con mutipa, que aparece como complemento de la preposición con. Podemos afirmar que mutipa es un sustantivo porque las preposiciones seleccionan sustantivos $\mathrm{u}$ oraciones como complemento. Además, con y sin pueden seleccionar nombres desnudos en singular, por lo que mutipa podría ser un sustantivo común contable o no contable. Aquí la ortografía nos permite descartar que se trata de un nombre propio. Si en lugar de mutipa, tuviéramos el plural mutipas, podríamos afirmar que se trata de un sustantivo común contable.

En el caso de brosqui, poquis y siquelición, reconocemos que son sustantivos comunes porque van precedidos por un artículo, que es una configuración sintáctica en la que aparecen los sustantivos para dejar de designar propiedades y funcionar como expresiones referenciales. Vemos, además, que brosqui es un nombre contable (un brosqui, dos brosquis, etc.), que poquis es plural (poqui) - por lo tanto, también es contable- y designa un conjunto no formado únicamente por entidades de género femenino (los poquis vs. las poquis) y que siquelición presenta el sufijo -ción, característico de los sustantivos deverbales. Grasca parece estar funcionando como un sustantivo no contable, ya que no está precedido por determinante y aparece en la forma de singular. Umpa tal vez sea un elemento que cuantifica la cantidad de grasca involucrada en el evento. Finalmente, nos queda Murolla, que por la ortografía podríamos indicar que es un nombre propio. Sin embargo, independientemente de la ortografía, sabemos que es un nombre propio por el contexto sintáctico en el que aparece: no está precedido por un artículo y está modificado de manera no restrictiva, lo que da la pauta de que designa una entidad particular (es una expresión referencial) ${ }^{5}$. Una actividad que se desprende de este análisis es reescribir la oración de (3) utilizando palabras del español, pero respetando en todos los casos las configuraciones sintácticas. Dejamos, a modo de ejemplo, una opción posible.

Con alegría, un cocinero preparó mucha comida y Pedro, a quien los huéspedes habían invitado, entonó la canción.

Nuestros estudiantes pueden "traducir" perfectamente un texto como el de (2) al español, sin manejar los conceptos de sustantivos, verbos, etc. Como docentes de lengua, entonces, debemos aprovechar esos conocimientos para profundizar en la reflexión metalingüística, pero para eso, nosotros debemos tener claro cuáles son las características fundamentales para determinar la pertenencia de una palabra a una clase particular. Lo que mostré con los sustantivos de la primera oración, puede realizarse con todas las palabras del texto y profundizar en mayor o menor medida en la reflexión gramatical. Pero además de esto, me interesa señalar que esa reflexión gramatical es posible sin conocer el significado de las palabras en juego, algo que

5 Quisiera hacer notar que del análisis del texto de (2) se desprende que la distinción entre sustantivos abstractos y concretos, siempre presente en la clasificación de sustantivos en el ámbito escolar y basada en criterios semánticos, carece de pertinencia gramatical, a diferencia de las distinciones contable-no contable y propio-común. 
evidentemente atenta contra la comunicación. En (2) no hay comunicación, no hay significado, pero hay gramática y, por esto, podemos reconocer clases de palabras (y subclases).

Los docentes de lengua debemos también ser conscientes de las falencias de las clasificaciones y de que hay ciertas palabras que, según el contexto en el que aparezcan, pueden pertenecer a más de una clase. En realidad, la pertenencia a una clase no es una propiedad inherente de una palabra, sino que se determina de acuerdo con los contextos en los que puede aparecer. Muchas veces, como ciertas palabras aparecen de manera sistemática en un contexto particular, tendemos a señalar que prototípicamente pertenece a una clase.

Para no perder el foco, sigamos con los sustantivos. Podemos decir que Emilia es un sustantivo propio en principio, ya que designa una entidad particular, es decir, constituye en sí mismo una expresión referencial. Sin embargo, Emilia puede aparecer en distintos contextos que vale la pena recuperar.

a. Emilia creció mucho.

b. La Emilia creció mucho.

c. La Emilia que se sentaba al fondo creció mucho.

En cuanto a la denotación, (5a) y (5b) son idénticas: designan una única entidad llamada Emilia. La diferencia está dada en términos de variación, ya que (5b) pertenece a alguna de las variedades del español que admiten el artículo con nombre propio, si hay una relación de amistad, familiaridad, cercanía. Por otro lado, $(5 \mathrm{c})$ desencadena la lectura de más de un referente al que se le aplica la denominación Emilia y, por lo tanto, son necesarias otras indicaciones (que se sentaba al fondo) para señalar un referente único. En este caso, el sustantivo Emilia no se comporta como propio, sino como común: la presencia del artículo es obligatoria y es modificado restrictivamente.

Para finalizar este apartado, me interesa volver a la cuestión de las preguntas que deberíamos hacernos los docentes para poder posteriormente motivar la reflexión gramatical en nuestros estudiantes. Con respecto a la clasificación de las palabras, es central preguntarnos cómo sabemos que una palabra es un verbo / sustantivo / adjetivo / adverbio; qué palabras parecen más difíciles de clasificar y por qué; y si dejando a un lado la comunicación es posible reconocer una gramática, como sucede en el texto de $(2)^{6}$. En el próximo apartado vamos a ver que no solo reconocemos clases de palabras en ese texto, sino que también podemos avanzar en discusiones vinculadas con la estructura argumental y las funciones sintácticas.

\section{Sobre la estructura argumental y las funciones sintácticas}

En el apartado anterior, planteamos que los criterios de clasificación de palabras que se proponen pueden ser diversos e incluir aspectos léxicos, ortográficos y morfosintácticos. Sin embargo, el criterio formal parece ser adecuado descriptivamente, ya que podemos reconocer la pertenencia de una palabra a una clase, sin conocer su significado. Los criterios

6 Una actividad para reflexionar sobre la distinción entre comunicación y gramática es presentar a continuación de un texto como el de (2) otro texto en el que se eliminen algunos elementos funcionales, pero en el que se empleen palabras que pertenecen al español. Por ejemplo: Chico limpiar casa y ordenar papeles. El contraste entre los dos tipos de textos puede llevar a discusiones sumamente interesantes con respecto a la gramática de una lengua, la comunicación y el significado. 
formales implican características morfológicas, como la denominada morfología flexiva, y la distribución de una palabra en un contexto sintáctico. Estos dos aspectos son centrales para las reflexiones que abordamos en este apartado. Veamos algunos ejemplos:
a. La pediatra trabajó toda la tarde.
b. Laura es pediatra.
c. Laura es la pediatra.

En las tres oraciones de (6) encontramos la palabra pediatra, a la que solemos clasificar como sustantivo. Si seguimos la definición que se propone sobre sustantivos comunes, pediatra es un sustantivo porque "nombra un ser de manera general y se escribe con minúscula"7. Sin embargo, esta definición no se aplica en (6b), ya que aquí pediatra refiere a una propiedad que se predica con respecto a Laura. Por otra parte, en (6a) y (6c) pediatra no nombra a un ser, sino que la referencia a un ser la hace el constituyente la pediatra, formado por el artículo y el sustantivo. Si volvemos a las discusiones desarrolladas en el apartado anterior, podemos indicar que pediatra es un sustantivo porque presenta información de género y número no derivada por concordancia con otro constituyente (6a) y porque para desempeñar la función de argumento -es decir, para identificar entidades del mundo extralingüístico-, necesita ser parte de una construcción más compleja nucleada por un determinante (Abney, 1987). De lo contrario, se tratará de un predicado $(6 \mathrm{c})^{8}$. En las próximas secciones nos detendremos en los diferentes aspectos de la relación entre predicados, argumentos y clases de palabras.

\subsection{Todas las clases de palabras pueden ser predicados}

En la introducción a este apartado, destacamos que un sustantivo puede funcionar como predicado. Evidentemente, esto entra en contradicción con las definiciones para esta clase de palabras y, además, pone de relieve una distinción que no está tan presente en la diferenciación entre sustantivos propios y comunes: los sustantivos propios no funcionan como predicados, en tanto que no atribuyen propiedades a ningún argumento, ni describen relaciones existentes entre argumentos. Comparemos las oraciones a continuación:
a. Laura es pediatra.
b. Laura es la pediatra.
c. Oscar es Emilio.

En (7a), pediatra es un predicado y como tal atribuye una propiedad (la de ser pediatra) a un argumento (Laura). La presencia del constituyente Laura se justifica a partir del predicado pediatra, que lo selecciona. En (7b) la pediatra designa una entidad al igual que Laura. Aquí no hay una relación de predicación, sino que encontramos dos constituyentes que identifican entidades y lo que indica esta oración es que, en ese universo discursivo, hay una única entidad: Laura y la pediatra refieren a la misma entidad. Si ponemos estos dos constituyentes en relación a un predicado como saludar, es imposible interpretar que hay una

\footnotetext{
7 Definición de sustantivo común en el manual Lengua. Practicas del lenguaje 5. En movimiento, Buenos Aires: Santillana (2014).

8 Entendemos que el predicado es aquel objeto lingüístico que "atribuye una propiedad a un argumento o describe la relación existente entre argumentos” (Di Tullio, 2005, p. 101).
} 
única entidad (Laura saludó a la pediatra). Esto mismo sucede con la oración de (7c), en la que hay involucrados dos sustantivos propios. Los sustantivos propios, a diferencia de los comunes, son expresiones referenciales en sí mismos, es decir, no precisan de la presencia de un determinante para identificar entidades y, por esta razón, no funcionarían como predicados ${ }^{9}$.

Un problema frecuente en la enseñanza de la gramática es la identificación de "ser predicado" con "ser un verbo". Esta confusión está motivada porque los verbos en español y en muchas otras lenguas se vinculan con la morfología flexiva obligatoria que permite definir una oración (tiempo, modo, aspecto y concordancia). Dada esta característica, cuando hay más de un predicado en una oración y uno de ellos es verbal, solemos reconocerlo como el predicado principal. Sin embargo, como docentes debemos distinguir estas dos nociones, ya que no todos los predicados son verbales, ni todos los verbos son predicados, como queda en evidencia en la oración de (7b): el predicado es el sustantivo pediatra, no el verbo ser. De hecho, la relación de predicación es totalmente independiente de la presencia de este verbo: Laura, pediatra.

Además de los verbos y los sustantivos, otras clases de palabras funcionan como predicados. Los adjetivos, indudablemente, atribuyen propiedades a un argumento (8a) o describen una relación entre argumentos (8b). Subrayo los argumentos seleccionados por el adjetivo orgulloso en cada caso.

a. Esteban es orgulloso.

b. Esteban está orgulloso de sus hijos.

El contraste entre (8a) y (8b) da lugar a unas cuantas reflexiones gramaticales que podemos trabajar con nuestros estudiantes. Una muy interesante es la distribución de los verbos copulativos ser y estar ${ }^{10}$. La presencia de uno u otro en el caso de (8) está en estrecha relación con la selección argumental del predicado orgulloso. Asimismo, la definición que sigamos para orgulloso en cada caso depende estrictamente de esa selección argumental. Es decir, el significado que se le atribuya a este adjetivo depende de la estructura en la que aparezca. Veamos otros casos en los que la estructura es fundamental para determinar el significado (ver discusión en Mateu, 2015).

(9) a. Juan le dio un libro a Estela.

b. Me da miedo la oscuridad.

c. Esa camisa da doctor.

Podríamos seguir agregando oraciones a la lista de (9), pero con estas tres es suficiente para plantear el problema. Es incorrecto postular que en las tres oraciones el predicado es el

9 Hay algunos usos de sustantivos propios (y pronombres) como predicados, que vale la pena trabajar con nuestros estudiantes para profundizar en la distinción entre predicados y argumentos. En las oraciones de (i) y (ii), los constituyentes Maradona y yo no designan entidades, sino una serie de propiedades.

(i) Esos tipos son Maradona.

(ii) Ese estilo es muy yo.

De hecho, el fenómeno de formación de verbos a partir de nombres propios está estrechamente vinculado a estos usos predicativos: icardiar no significa ser Icardi (el jugador de fútbol), sino presentar una conducta relacionada a una serie de propiedades vinculadas con alguna característica de este jugador de fútbol (ver Mare y Silva Garcés, 2018, para una descripción general de estos casos).

10 Para la distribución de los verbos copulativos ser y estar ver Brucart (2010), Mugica y Mangialavori Rasia (2012), entre otros. 
verbo dar. Por ejemplo, en (9a) la predicación de transferencia está determinada por las combinaciones en esa estructura (algo a alguien). En (9b), los argumentos que aparecen (me refiriendo a la primera persona de singular y la oscuridad) no son seleccionados por dar, sino por dar miedo. Con respecto a (9c), también podríamos indicar que la predicación en relación al argumento esa camisa es dar doctor. Ni miedo ni doctor son argumentos, sino que funcionan como predicados en combinación con el verbo $d a r^{11}$.

Para terminar con los adjetivos, vale destacar que además de ser predicados, pueden funcionar como argumentos, y este aspecto es relevante para subclasificaciones como ser un adjetivo calificativo o relacional. Dentro de los adjetivos relaciones reconocemos el subgrupo de los argumentales, ya que parecen introducir los argumentos de un sustantivo. Esto es lo que se observa en los sintagmas la pesca ballenera y las invasiones inglesas, que pueden parafrasearse como pescar ballenas y los ingleses invaden, respectivamente.

Finalmente, me interesa detenerme en las preposiciones, elementos relacionales por naturaleza y, por lo tanto, predicados. Sin embargo, al igual que lo que sucede con los verbos, no todos los ítems que se clasifican como preposiciones funcionan como predicados. Comparemos, por ejemplo, la preposición de con la preposición sobre.

a. El padre de Ana.

b. La pesca de ballenas.

c. Juan está orgulloso de sus hijos.

a. El libro está sobre la caja.

b. Juan puso el libro sobre la caja.

En los casos de (10), la presencia de los argumentos Ana (10a), ballenas (10b) y sus hijos (10c) no se vincula con el ítem de, sino con los predicados padre, pesca y orgulloso, respectivamente. Este elemento aparece por cuestiones estrictamente formales de la gramática del español, no por razones de selección argumental. De hecho, tanto en (10a) como en (10b) es sustituible por otras formas, sin que cambie el sentido del predicado padre y ballena: su padre; la pesca ballenera.

La preposición sobre, en cambio, indica un tipo de relación entre dos argumentos, relación que no se deriva de otros predicados involucrados (como poner en (11b)). En (11a) es claro que sobre establece una relación entre el argumento el libro y el argumento la caja, porque la presencia de ambos argumentos está determinada únicamente por la preposición: estar también es un verbo copulativo. En (11b) también se reconoce esta misma relación, pero puede ser menos clara por la presencia de puso, que es el predicado principal y establece una relación entre un agente, que desencadena el movimiento (Juan), un objeto desplazado (el libro) y un lugar (la caja). Sin embargo, la relación que se establece entre el libro y la caja, no está definida por poner, sino por sobre. Poner selecciona un lugar, pero la preposición (o un adverbio locativo) determina cuál es la relación entre el objeto desplazado y ese lugar: sobre, en, bajo, ahí, dentro, atrás, etc. Además, el argumento que indica lugar, depende estrictamente de la preposición / adverbio locativo, ya que si nuestra oración fuera Juan puso el libro dentro de la caja, sería imposible reemplazar la caja por la mesa (?Juan puso el libro dentro de la mesa).

11 En niveles más avanzados vale la pena reflexionar sobre los sustantivos desnudos que también aquí funcionan como predicados o parte de predicados, como ya vimos en Laura es pediatra. 
Esta imposibilidad es de índole semántica y no tiene que ver con el significado de poner-dado que Juan puso el libro sobre la mesa es perfecta-, sino con el significado del adverbio y del constituyente la mesa.

En resumen, las reflexiones en torno a las posibilidades de predicación de las distintas clases de palabras permiten profundizar en diversos contenidos gramaticales y recurrir a los conocimientos que tienen nuestros estudiantes como hablantes nativos de una lengua. El docente puede graduar las discusiones que proponga, de acuerdo con el nivel educativo en el que esté enseñando. Además, estos análisis que aplicamos a nivel oracional, pueden realizarse a nivel discursivo, ya que las relaciones entre predicados y argumentos son determinantes para la coherencia textual.

\subsection{La clasificación de los verbos en cuanto a su estructura argumental}

La clasificación de los verbos en transitivos e intransitivos surge de la relación entre predicados, argumentos y clases de palabras, y también constituye un contenido presente en el aula de lengua. Cuando abordamos la transitividad, entran en juego las funciones sintácticas, ya que para calificar un verbo como transitivo no basta con que seleccione dos argumentos, sino que uno de ellos debe cumplir la función sintáctica de objeto directo. Me interesa revisar algunas oraciones, adaptadas a partir del texto de (2), para avanzar en la discusión. Subrayo los verbos.

a. Murolla drinó la siquelición.

b. El brosqui tataba de poritación.

c. Zozó no cucó.

d. A la rutica le patiló la ratileza.

e. Zozó, Murolla y fitos brosquis cafaron para lastrofar la matuca.

Sin conocer el significado y reparando únicamente en las combinaciones que aparecen y en el conocimiento formal de clases de palabras que nos permitieron identificar argumentos, podemos clasificar todos los verbos de (12). Drinar es transitivo, tatar es intransitivo y tiene un complemento preposicional, cucar es intransitivo, patilar es intransitivo, pero no es igual que tatar ni que cucar, cafar es intransitivo y lastrofar es transitivo. Algunos que reconocimos como intransitivos, por ejemplo, cucar y cafar, podrían ser transitivos que admiten objetos nulos. Para definir esto necesitaríamos observar cómo funcionan en otros contextos. Es decir, necesitaríamos contar con más datos. Considerando solamente la oración de (12c), no sabemos tampoco si cucar, como intransitivo, es inergativo o inacusativo, es decir, si el único argumento (Zozó) es un agente o es un tema. Pronto volveremos sobre estos puntos.

Lo que resulta interesante es que todas las oraciones de (12) pueden ser analizadas sintácticamente. Es decir, puede suceder que necesitemos más datos para definir con certeza los roles temáticos de los argumentos involucrados, pero con la información presente en (12), es posible determinar sujetos, objetos directos y otras funciones. Veamos:

(13) a. Murolla SUJETo drinó la siquelición $_{\text {OBJETo DIRECTO }}$

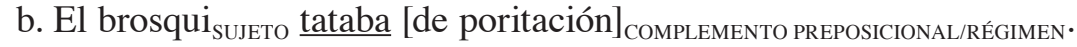

c. Zozó ${ }_{\text {SUjeto }}$ no cucó.

d. [A la rutica $]_{\text {DATIVo de interés }}$ le patiló la ratileza SUJeto $_{\text {. }}$

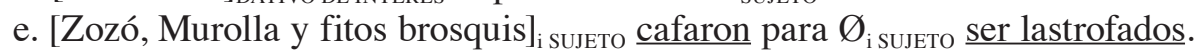


Lo que me interesa hacer notar con este juego es que ninguna de las definiciones semánticas a las que se suele recurrir para identificar funciones sintácticas ("el sujeto es el que realiza la acción", por ejemplo), sirve para analizar sintácticamente las oraciones de (12) y, aun así, es posible realizar el análisis. No podemos saber si el sujeto realiza alguna acción, porque desconocemos el significado de los verbos: drinar podría significar ordenar y en ese caso el sujeto sí realizaría una acción, pero también podría significar sentir y no habría ninguna acción involucrada. Sin más datos, no podemos conocer los roles temáticos (agente, tema, experimentante), pero sí identificar las funciones sintácticas. ¿Cuáles son las marcas? La concordancia con el verbo para los sujetos (el brosqui SG $_{\mathrm{S}}$ tataba $_{\mathrm{SG}}$ vs. los brosquis PL $_{\mathrm{PL}}$ tataban $_{\mathrm{PL}}$; le patiló SG $_{\mathrm{SG}}$ ratileza $a_{\mathrm{SG}}$ vs. le patilaron PL $_{\mathrm{PL}}$ las ratilezas $\mathrm{PL}_{\mathrm{PL}}$; la sustitución por el clítico acusativo (lo / la / los / las) para los objetos directos (Murolla la drinó); el doblado por el clítico dativo para los distintos dativos (a la rutica le patiló), además de la presencia de la preposición a (a la rutica).

Otra marca formal es el ítem de que media entre tataba y la poritación en (13b). Este elemento indica claramente que la poritación no es un objeto directo. Finalmente, en (13e) encontramos el constituyente para Ø ser lastrofados, en el que aparece la forma pasiva del verbo lastrofar. Asumimos que hay un elemento que no se pronuncia $\varnothing$, ya que ser lastrofado es un predicado que requiere de un argumento. Ese argumento, no aparece explícito, pero se interpreta a partir de un antecedente (Zozó, Murolla y fitos brosquis). Sabemos que ese argumento, que no tiene contenido fonológico, es un sujeto, dado que las estructuras pasivas se caracterizan por el hecho de que el argumento que conservan es el que establece la concordancia y, por lo tanto, es el sujeto -independientemente de que cumpla la función sintáctica de objeto directo en la oración activa (Ellos lastrofaron a Zozó, Murolla y fitos brosquis $>$ Ellos los lastrofaron). En consecuencia, hay marcas formales -la voz pasiva- que nos permite reconocer que lastrofar es un verbo transitivo.

Volvamos ahora al vocabulario conocido del español y revisemos las oraciones de (14). En (14a) encontramos un verbo transitivo, en (14b) un verbo intransitivo inergativo y en (14c) un verbo intransitivo inacusativo.
a. Juan ordenó los libros.
b. Juan caminó.
c. Llegó Juan.

Ya vimos que no necesitamos conocer el significado para definir esta información, por lo que es interesante hacer el ejercicio con nuestros estudiantes de argumentar desde los aspectos formales. (14a) es transitivo, porque presenta un argumento que funciona como sujeto (Juan) y un argumento que funciona como objeto directo (los libros). De Juan se predica que ordenó los libros y si queremos formar una oración absoluta, podemos dejar afuera a Juan (ordenados los libros), pero no a los libros (*ordenado Juan, con la interpretación de 'Juan ordenó'). Esto indica que la relación con el argumento los libros es más estrecha, de ahí que se plantee que es el argumento interno, mientras que Juan es el argumento externo ${ }^{12}$. Esta relación es formalizable en una estructura arbórea como la de (15), en la que el Argumento 1 representa el argumento externo y el Argumento 2 el interno.

12 Hay otras pruebas formales que muestran que la relación entre el verbo y el argumento interno es más estrecha que entre el verbo y el argumento externo. Ver Bosque y Gutiérrez-Rexach (2009), capítulo 6. 
(15)

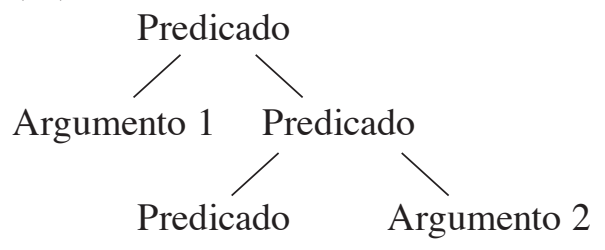

Con esta idea en mente e independientemente del esquema de (15), se puede analizar la relación entre el verbo y el único argumento que encontramos en (14b) y (14c). En ambos casos, este argumento cumplirá la función sintáctica de sujeto, sin embargo, al intentar formar una oración absoluta, vemos que hay diferencias entre ellos. En (14b) la oración absoluta da un resultado tan anómalo como cuando intentamos construirla con el argumento externo de ordenar: *caminado Juan, se dio un baño. En (14c), en cambio, la construcción absoluta es tan buena como cuando la construimos con el argumento interno de ordenar: llegado Juan, empezó la fiesta. Por esta razón, se proponen estructuras diferentes para estos dos tipos de verbos intransitivos. En el caso de los inergativos, se reconoce un argumento externo, ensamblado en una posición como la del Argumento 1 en (15), mientras que en los inacusativos se propone un argumento interno, ensamblado en una posición como la del Argumento 2 en (15) ${ }^{13}$.

Ahora bien, volviendo a los verbos aparentemente intransitivos de (12), podemos proponerles a nuestros estudiantes que sugieran qué tipos de datos deberíamos encontrar para poder definir si son transitivos, inacusativos o inergativos. Pensemos en cucar (Zozó no cucó). Si en otros contextos encontramos algo como Zozó cucó una gridata, podemos concluir que se trata de un verbo transitivo que admite objetos nulos (del tipo de comer, fumar, beber, leer, escribir). Esperaríamos, entonces, encontrar expresiones como cucada la gridata o una gridata recién cucada, pero no cucado Zozó. Si en el rastreo de datos, no encontramos nada como Zozó cucó una gridata, ni como cucado Zozó podremos concluir que cucar es un verbo inergativo. Ahora bien, si no hay datos de transitividad, pero sí hubiera ejemplos como recién cucado Zozó, podemos indicar que estamos ante un verbo inacusativo. Vemos nuevamente que el juego con palabras inventadas obliga a recurrir a pruebas formales que nos permiten avanzar en la reflexión gramatical.

Antes de finalizar esta sección, es importante relacionar la clasificación verbal en cuanto a la transitividad con un aspecto del discurso, como es la estructura de la información. En (14) podemos prestar atención al orden en el que aparecen los distintos constituyentes: (14a) SUJETO-VERBO-OBJETO; (14b) SUJETO-VERBO y (14c) VERBO-SUJETO. Luego de la discusión con respecto a los tipos de argumentos y a la relación entre el argumento de los verbos intransitivos y los argumentos de los verbos transitivos, no resulta extraño que (14b) y (14c) presenten posiciones diferentes para su sujeto. En (14) seguimos el orden no marcado, es decir, el que se corresponde con la estructura argumental. Cualquier modificación a este orden supone algún tipo de efecto discursivo, como podría ser la tematización o la focalización de un constituyente. Asimismo, cualquier omisión de un argumento es relevante a los fines de la comunicación. Veamos algunas opciones para (14a):

13 Esta discusión se inscribe en la teoría de la estructura argumental desarrollada por Hale y Keyser (1993; 2002) y en otras aproximaciones sintáctico-céntricas como la de Mateu (2002), Harley (2011), Marantz (2013), entre otros. 

a. Juan ordenó los libros. (orden no marcado)
b. Ordenó los libros. (omisión del sujeto)
c. Juan los ordenó. (omisión del objeto)
d. Los ordenó. (omisión del sujeto y del objeto)
e. Con respecto a Juan, ordenó los libros. (tematización del sujeto)
f. En cuanto a los libros, Juan los ordenó. (tematización del objeto)
g. Hablando de ordenar, Juan ordenó los libros. (tematización del predicado)
h. Los libros ordenó Juan. (focalización del objeto)

Lo interesante de las oraciones de (16) es que en todos los casos la predicación es la misma (Juan ordenó los libros), sin embargo, la estructura informativa varía. Poder identificar el orden no marcado es central para reconocer qué procesos tuvieron lugar a nivel informativo: ¿qué información le interesa destacar a nuestro interlocutor?, ¿qué información omite y por qué?, ¿cómo se relaciona ese enunciado con el resto del discurso? Finalmente, veamos qué sucede con las omisiones si en lugar de un sujeto de tercera persona, como Juan, tenemos un sujeto de primera persona del singular.
a. Juan ordenó los libros.
b. Ordenó los libros.
a. Yo ordené los libros.
b. Ordené les libros.

Las marcas formales vinculadas con la concordancia verbal (ordenó vs. ordené) son centrales para definir que en (17a) la presencia del sujeto es informativa y si se omite es porque se trata de información que se asume como conocida por nuestro interlocutor. Ordenó puede tener infinidad de referentes como sujeto, algo que no sucede con ordené, que solo puede referir al hablante. Por esta razón, (18a) con el pronombre expreso es una construcción marcada, que incluso podría interpretarse como un tipo de reproche, interpretación imposible para (18b). Evidentemente, estas nociones son fundamentales para la producción e interpretación de discursos. De hecho, lo que realizamos con oraciones creadas a los fines de esta presentación, podemos trasladarlo fácilmente a textos reales, como el de (19).

(19) La noté rara, o diría: ansiosa. Como quien teme algo, algún acontecimiento desagradable que, de todos modos, va a sobrevenir. Le pregunté qué le pasaba. Con agresividad dijo que no le pasaba nada (Castillo, 1992, p. 15).

En (19) aparecen las cuatro oraciones que dan inicio al cuento "Capítulo para Laucha". Lo primero que llama la atención es el empleo de clíticos de tercera persona, marcados en negrita, que dan información de un referente femenino singular, pero que no podemos recuperar. En relación con la discusión sobre (17) y (18), vemos el contraste entre la morfología de tercera persona y la de primera persona del singular, manifestada en la flexión verbal y que refiere únicamente al hablante. Es decir, en ambos casos no se explicita el argumento por medio de una expresión referencial plena (fonológica y semánticamente), sin embargo, hay una diferencia notable que, en este caso, busca producir un efecto en el lector. Si se compara este fragmento con el inicio de un texto académico, será fácil notar la diferencia entre los tipos textuales, con respecto a la omisión de referentes, orden de palabras y otros aspectos estrictamente gramaticales. 


\subsection{Argumentos que se agregan, se borran o se degradan}

El último aspecto que quisiera tratar con respecto a la relación entre predicados y argumentos es el que se vincula con las alteraciones en la estructura argumental "esperable". En el apartado anterior me detuve en los tipos de verbos en cuanto a su transitividad a partir de la definición en tres clases: transitivos, inergativos e inacusativos. En este apartado me interesa ver cómo en los usos de los hablantes, estas estructuras pueden verse alteradas, sin que ello resulte de una arbitrariedad impredecible, sino más bien, de procesos definidos y productivos. Evidentemente, este es un aspecto que merece un espacio en el aula de lengua. Veamos algunos ejemplos.
a. Al herido lo llegaron al hospital unos vecinos.
b. Rafael no murió... lo murieron los gendarmes.
c. A los documentos los desapareció la secretaria.
d. Al avión lo cayeron los norteamericanos.
a. A Estercita la re caminaron los de la ART.
b. River lo madrugó a Boca en el último clásico.
c. El Barça bailó al Real Madrid.
d. Estercita me está vacilando.

En (20) encontramos verbos que han sido calificados como inacusativos, mientras que en (21) aparecen inergativos. Sin embargo, todas las oraciones de (20) y (21) son transitivas, ya que reconocemos un sujeto y un objeto directo. Evidentemente, hay un argumento que se agregó a la estructura, no obstante, los resultados de este agregado no son los mismos y esto parece tener relación con el tipo de verbo intransitivo en juego (ver Pujalte y Zdrojewski, 2013). En los casos de (20), el argumento subrayado se interpreta como un agente que provoca que el evento suceda, es decir, que el herido llegue al hospital, que Rafael muera, que los documentos desaparezcan y que el avión caiga. Es decir, en todos los casos, recuperamos el predicado que selecciona un argumento interno, algo ya propio de los verbos inacusativos, y se agrega el argumento externo, resultando en una estructura como la de (15).

En (21), en cambio, estas lecturas son imposibles: no es que aparezca un agente que hace "caminar a Estercita", "madrugar a Boca", "bailar al Real Madrid" o "vacilar al hablante". En todos estos casos hay un significado nuevo, que no se deriva composicionalmente y que, por lo tanto, debe ser aprendido. Ambas listas de oraciones pueden seguir aumentando y, seguramente, serán nuestros estudiantes quienes nos brinden más datos. Nuestro rol como docentes es, entonces, proponerles un espacio de reflexión metalingüística, en el que puedan analizar estos datos considerando otros temas abordados en clase.

Hay un caso más de argumentos agregados que resulta interesante para abordar en el aula, ya que va de la mano con una problemática de discusión actual, como es el uso machista de la lengua.
a. Te lavo los platos.
b. Juan me limpió los pisos.
c. Mirame un minuto al bebé. 
En (22) se agrega un argumento a una estructura transitiva y se dice que ese argumento es un dativo no argumental, ya que el predicado verbal no lo selecciona. La presencia de este elemento tiene claras consecuencias discursivas, porque establece algún tipo de relación de posesión o de beneficio para la entidad introducida por el dativo (ver Pineda (2013) y Pujalte (2009), para la introducción de otros argumentos por medio de núcleos aplicativos en español). Las reflexiones sobre los usos lingüísticos machistas pueden desencadenar el abordaje sobre este tipo de ejemplos ${ }^{14}$.

Otro cambio en la estructura argumental consiste en el borrado de argumentos, perspectiva desde la que podemos discutir con nuestros estudiantes muchas de las construcciones denominadas pronominales. Observemos los datos a continuación.
a. Juan se peinó.
b. El vaso se rompió.
c. Se trabaja mucho acá.
d. Se eligieron representantes.
e. Los chicos se ayudaron.

En la introducción recuperé la siguiente frase de Di Tullio (2005, p. 103): “el predicado es como el guión de una obra: determina cuántos participantes se requieren y asigna a cada uno un papel". Al mirar los ejemplos de (23) bajo esta metáfora, podríamos postular que el predicado efectivamente determina los participantes y sus roles, pero el se constituye una marca de "película de bajo presupuesto", en la que, para reducir costos, se omite la presencia de un participante. Evidentemente, esta descripción no cubre todos los casos en los que aparece el clítico se en español (ver Bosque y Gutiérrez-Rexach, 2009, §6.11), pero resulta relevante para incluir la discusión de estas formas en las reflexiones sobre la estructura argumental, ya que en muchas de ellas (todos los ejemplos de (23) y similares) la presencia del clítico marca la ausencia de un argumento; y es precisamente esa ausencia, en combinación con las propiedades del predicado, la que habilita las interpretaciones relevantes: reflexividad (23a), anticausatividad (23b), impersonalidad (23c), pasividad -y también impersonalidad- (23d), reciprocidad (23e). Por esta razón, resulta siempre relevante recuperar las construcciones correspondientes sin el clítico, para entender qué está sucediendo en oraciones como las de (23) y reconocer que, si bien hay un argumento que se borra, el que permanece mantiene su papel temático.
a. Juan peinó a su hija.
b. El golpe rompió el vaso.
c. Los empleados trabajan mucho acá.
d. Los estudiantes eligieron representantes.
e. Los chicos ayudaron a sus amigos.

Abordar este tipo de construcciones de esta manera, es decir, considerando que hay un argumento que falta (Embick, 2004; Pujalte y Saab, 2012), resulta relevante no solo para avanzar en la reflexión gramatical, sino también para el análisis de diferentes discursos y para

14 De hecho, hay un chiste de la humorista argentina Maitena, que se basa en este tipo de dativos agregados en vinculación con el machismo en las lenguas. 
la reflexión sobre la escritura de textos académicos: ¿qué participante se borra?, ¿cómo se interpreta la ausencia de ese participante?

Finalmente, consideremos las construcciones pasivas perifrásticas, en las que hay una alteración en la estructura argumental, pero no como resultado del borrado de un argumento, sino como consecuencia de su "degradación”. Siguiendo la metáfora de los guiones, podríamos considerar que un participante que tenía el papel principal (ser argumento externo y cumplir la función sintáctica de sujeto), pasa a desempeñar un papel secundario (ser parte de un sintagma preposicional encabezado por la preposición por), tan secundario que incluso puede omitirse. Sin embargo, esta degradación si bien afecta las funciones sintácticas, no altera el rol temático del argumento interno. De ahí la relevancia de distinguir entre funciones sintácticas y roles temáticos ${ }^{15}$. También, vale decir aquí, que esta presentación no agota en absoluto el tema de las construcciones pasivas, pero sí resulta relevante introducirlo en relación con la estructura argumental ${ }^{16}$.

(25) a. Los representantes fueron elegidos (por los estudiantes).

b. Rafael fue asesinado (por la gendarmería).

c. Fuimos invitados a un congreso (por los organizadores).

a. Los estudiantes eligieron a los representantes.

b. La gendarmería asesinó a Rafael.

c. Los organizadores nos invitaron a un congreso.

Nuevamente, estas discusiones son relevantes para el análisis de textos, ya que se vinculan estrechamente con la estructura de la información presentada (temas y remas). Si consideramos con Halliday (1967) que el tema o tópico es "aquello de lo que se habla, o el punto de partida de la oración", podemos entender la diferencia entre (25) y (26) en relación con los recursos de tematización: el tema en (25a) es el constituyente los representes, mientras que en (26a) es el constituyente los estudiantes. Podemos agregar estos datos a los que presentamos al final de $\$ 4.2$, para avanzar en los aspectos discursivos.

En resumen, a lo largo de esta sección revisé una serie de datos que pueden abordarse a partir de la relación entre predicados y argumentos. Uno de los temas relevantes para incorporar en el aula de lengua es el agregado, el borrado y la degradación de argumentos, que, además de discutirse desde perspectivas formales, resulta interesante para trabajar en relación con la producción y análisis de discursos. Por supuesto, cada uno de los fenómenos presentados tiene sus propias líneas de investigación y profundización que exceden lo relativo a la estructura argumental. Sin embargo, podemos volver una y otra vez sobre estos desde diferentes ángulos, en virtud de los contenidos y objetivos que deseemos abordar con nuestros estudiantes.

\section{Consideraciones finales}

A lo largo de estas páginas, presenté una serie de temas y discusiones que deben ser considerados para promover el pensamiento crítico y el desarrollo de habilidades lingüísticas

15 Recordemos nuevamente la definición de sujeto como el que realiza la acción. Las oraciones pasivas constituyen un claro contraejemplo a esta definición que mezcla funciones sintácticas con roles temáticos.

16 Un tema muy interesante para trabajar con los estudiantes de niveles superiores es la diferencia entre las construcciones pasivas con el clítico se (24d) y las pasivas perifrásticas (25a). 
en los diferentes niveles educativos. Hay nociones que no pueden estar ausentes en la elaboración de un programa y de las actividades de lengua, como las de variación lingüística, gramaticalidad y registro. Este último concepto es parte de una diferenciación que estuvo presente a lo largo del texto: la distinción entre lo comunicativo y lo formal. Con respecto a los temas específicos que abordamos, intentamos destacar la vinculación entre clases de palabras, estructura argumental y funciones sintácticas, como contenidos presentes en los diseños curriculares y que pueden abrir un amplio panorama para proponerles a nuestros estudiantes un estudio científico de la lengua.

En relación con este aspecto, cabe reflexionar sobre el lugar que le damos a las etiquetas en la enseñanza: ¿lo importante es la etiqueta que podamos poner o la discusión que subyace a la clasificación de los "objetos" del lenguaje y que muchas veces nos lleva a concluir que es difícil colocar una etiqueta? Por momentos, parece que el objetivo de la enseñanza de la gramática fuera colocar una etiqueta y justificarla como sea. Esto puede llevarnos a dejar a un lado la reflexión gramatical a partir de la consideración de datos que efectivamente están presentes en la lengua. No debemos olvidar que las etiquetas son constructos humanos y, como tales, pueden ser discutidas, repensadas y reformuladas.

Para poder pensar un abordaje de la gramática en estos términos, es fundamental la formación continua, por un lado, y la actitud científica, por el otro. Por actitud científica nos referimos a no asumir los conocimientos como acabados, sino hacernos preguntas y motivar a nuestros estudiantes para que también se hagan preguntas. Y aquí queremos hacer hincapié en "se hagan preguntas" contra "nos hagan preguntas". Una actitud científica no resulta de buscar que otros nos brinden respuestas, sino en tratar de encontrarlas en el trabajo reflexivo y colaborativo. Intentar llegar a esto en el aula de lengua no es un objetivo descabellado: tenemos muchos científicos en potencia que, como hablantes nativos de una lengua, tienen muchísimo para decir, discutir y aportar.

\section{Bibliografía}

Abney, S. (1987). The English noun phrase in its sentential aspect. (Tesis de doctorado). Massachusetts Institute of Technology.

Bertolotti, V. (2014). Pronombres posesivos en el español rioplatense: tres casos de reanálisis. Traslaciones. Revista Latinoamericana de Lectura y Escritura, 1(1), 56-74.

Bosque, I. y Gutiérrez-Rexach, J. (2009). Fundamentos de sintaxis formal. Madrid: Akal.

Brucart, J. M. (2010). La alternancia ser y estar y las construcciones atributivas de localización. En A. Avellana (Ed.), Actas del V Encuentro de Gramática Generativa (pp. 115-152). Neuquén: EDUCO.

Carrió, C. (2014). Lenguas en Argentina. Notas sobre algunos desafíos. En L. Kornfeld (Comp.), De lenguas, ficciones y patrias (Cuadernos de la Lengua) (pp. 149-184). Buenos Aires: Universidad Nacional General Sarmiento.

Castillo, A. (1992). Capítulo para Laucha. En A. Castillo, Cuentos crueles (pp. 15-22). Buenos Aires: EMECÉ Editores.

Di Tullio, Á. (2005). Manual de Gramática del Español. Buenos Aires: La Isla de la Luna. 
Embick, D. (2004). Unaccusative Syntax and Verbal Alternations. En A. Alexiadou, E. Anagnostopoulou y M. Everaert (Eds.), The Unaccusativity Puzzle: Explorations of the Syntax-Lexicon Interface (pp. 137-158). Oxford: Oxford University Press.

Hale, K. y Keyser, S. J. (1993). On argument structure and the lexical expression of syntactic relations. En K. Hale y S. J. Keyser (Eds.), The View from Building 20: Essays in Linguistics in Honor of Sylvain Bromberger (pp. 53-109). Cambridge, Massachusetts: MIT Press.

Hale, K. y Keyser, S. J. (2002). Aspect and the Syntax of Argument Structure (ms.). Cambridge, Massachusetts: Massachusetts Institute of Technology.

Halliday, M. (1967). Notes in transitivity and theme in English. Journal of Linguistics, 3, 199-243.

Harley, H. (2011). A Minimalist Approach to Argument Structure. En C. Boeckx (Ed.), The Handbook of Linguistic Minimalism (pp. 427-448). Oxford y Nueva York: Oxford University Press.

Kornfeld, L. y Kuguel, I. (2013). Un afijo re loco. En Á. Di Tullio (Coord.), El español de la Argentina: estudios gramaticales (pp. 15-36). Buenos Aires: Eudeba.

Makuc Sierralta, M. (2010). La noción de significado en Frege, Russell y Wittgensttein: Aproximación filosófica comunicativas del lenguaje. Logos: Revista de Lingüística, Filosofía y Literatura, 20(2), 42-55.

Marantz, A. (2013). Verbal argument structure: Events and participants. Lingua, 130, 152-168.

Mare, M. (2014). El discurso científico. La argumentación en gramática. En A. Frischknecht (Coord.), ACORDE-MOS-NOS. De opiniones y ámbitos del hacer social (pp. 94-104). Buenos Aires: Planeta color ediciones.

Mare, M. (2015). Proyecciones funcionales en el ámbito nominal y concordancia. Un abordaje en términos de variación. Munich: Lincom.

Mare, M. y Silva Garcés, J. (2018). Estudiar las lenguas. En M. Mare y F. Casares (Eds.), ¡A lingüistiquearla! (pp. 23-44). Neuquén: EDUCO.

Mateu, J. (2002). Argument Structure. Relational Construal at the Syntax-Semantics Interface. (Tesis doctoral). Universitat Autònoma de Barcelona. Recuperado de https://www.tdx. cat/handle/10803/4828\#page=1

Mateu, J. (2015). La estructura argumental. En Á. Gallego (Ed.), Perspectivas de sintaxis formal (pp. 111-148). Madrid: Akal.

Ministerio de Educación, Ciencia y Tecnología de la Nación. (2006). Núcleos de aprendizajes prioritarios. Buenos Aires: Ministerio de Educación, Ciencia y Tecnología.

Mugica, N. y Mangialavori Rasia, M. E. (2012). A propósito de ser/estar: Reflexiones en relación a la instanciación gramatical de distintas expresiones de estado en español. Signo y Seña, 22, 105-118.

Pineda, A. (2013). Double object constructions and dative/accusative alternations in Spanish and Catalan: A unified account. Borealis. An International Journal of Hispanic Linguistics, 2(1), 57-115. 
Pujalte, M. (2009). Condiciones sobre la introducción de argumentos: el caso de la alternancia dativa en español. (Tesis de maestría). Universidad Nacional del Comahue.

Pujalte, M. y Saab, A. (2012). Syncretism as PF repair: the case of SE insertion in Spanish. The End of Argument Structure? Syntax and Semantics, 38, 229-260.

Pujalte, M. y Zdrojewski, P. (2013). Procesos de transitivización en el español del Río de la Plata. En Á. Di Tullio (Coord.), El español de la Argentina: estudios gramaticales (pp. 37-58). Buenos Aires: Eudeba.

Santillana. (2014). Lengua. Practicas del lenguaje 5. En movimiento, Buenos Aires: Santillana

Serra, R. y Caballer, M. J. (1997). El profesor de ciencias también es profesor de lengua. Alambique: Didáctica de las Ciencias Experimentales, 4(12), 43-49. 
